\title{
cDNA cloning and analysis of alaninerich cuticle protein gene of common fouling barnacle, Balanus amphi trite
}

\author{
Chandrakant B. Jagtap, Om Kinker Tiwari, Pradeep Kumar \\ Naval Materials Research Laboratory, Ambernath, Maharashtra, India.
}

\begin{tabular}{l}
\hline ARTICLE INFO \\
\hline Article history: \\
Received on: 16/03/2015 \\
Revised on: $31 / 03 / 2015$ \\
Accepted on: 22/04/2015 \\
Available online: 20/05/2015 \\
\hline Key words: \\
cuticular proteins, barnacle, \\
Balanus amphitrite, AAP(V/L)
\end{tabular}

\begin{abstract}
Hard cuticle of insects is a complex structure composed mainly of several proteins and chitin fibers. Cuticular proteins are important as presence of different cuticular proteins confer different mechanical properties to the exoskeletons of insects. Hence, there is growing interest in studying cuticular proteins as bioengineering of these proteins may help in synthesis of biomaterials for various applications. Till date many cuticle proteins have been identified from insects. These proteins show presence of characteristic motifs in them such as R\&R motif, extended R\&R motif, AAP (V/L) motif etc. We have identified one of such putative cuticle protein encoding gene from common fouling barnacle, Balanus amphitrite by cDNA cloning and sequencing. The gene was found to encode an open reading frame (ORF) of 102 amino acids. The deduced protein sequence was found rich in alanine forming almost $22 \%$ of the total amino acids. Other two predominant amino acids, valine and proline together contributed $\sim 23 \%$ of total amino acids. The hydropathy plot of the amino acid sequence showed presence of two hydrophobic regions surrounding central hydrophilic region. The protein sequence showed presence of three characteristic YHAAP (V/L) motifs at the C-terminus.
\end{abstract}

\section{INTRODUCTION}

Cuticle forms a barrier between animal and environment and thus protects it from outside environment. Cuticle is mainly composed of proteins that are embedded in chitin fibers. Hundreds of genes encoding for cuticular proteins have been identified in more than 20 species of insects, e.g. in silkworm genome more than two hundred genes encoding for cuticular proteins were identified, comprising almost $1.5 \%$ of the total predicted genes [1]. It has been observed that the composition of cuticle varies between different developmental stages of the same species [2-4]. Cuticular proteins are characterised by presence of $R \& R$ motifs in them which were first identified by [5]. $R \& R$ consensus sequence is conserved across arthropods, although its location and flanking residues vary considerably. This consensus sequence always begins with a triad of aromatic residues (Y/F-X-Y/F/W-XY/F) and ends with conserved G-F/Y amino acids. It has been recently observed that the extended $\mathrm{R} \& \mathrm{R}$ motif is actually involved in binding of proteins to chitin [6-7].

Many cuticular proteins have been identified without presence of an R\&R motif in them. However, these proteins have been found to have high content of alanine residues [8] and the overall protein sequence is dominated by hydrophobic amino acids. Such proteins have been reported in Locusta migratoria, Drosophilla melanogaster and Tenebrio molitor [8-10].
These proteins with high alanine content have AAP $(\mathrm{V} / \mathrm{L})$ motifs present in them. Although many cuticular proteins and genes have been identified in many insects, nothing is known about cuticular proteins of Barnacles.

Barnacles, also known as cirrepeds, are marine crustaceans classified under subclass Cirripedia of phylum Arthropoda. Moulting in barnacles is similar to other crustaceans except it happens beneath the hard shells. The cyprid larvae of Barnacle is covered with carapace (cuticle) which is shed after its settlement on the substrates.

The cyprids then turns into juveniles which finally metamorphose in to an adult. Adult has its cuticle that lies beneath the calcareous shell of the barnacle. Barnacle cuticle has not been studied yet and therefore, no information is available about proteins present in the barnacle cuticle. Only one entry of cuticular protein of Balanus amphitrite with PF00379 motif is present in Cuticle DB (http://bioinformatics.biol.uoa.gr/ cuticleDB/). In this report, we describe isolation and characterization of a cDNA clone (BCP-2) containing a gene encoding for alanine rich cuticle protein of Balanus amphitrite.

\section{MATERIALS AND METHODS}

\subsection{Animals/Organisms}

Adult barnacles of Balanus amphitrite were collected from Mudh island, Mumbai. Settled barnacles were brought to the laboratory and processed further for nucleic acid isolation.

* Corresponding Author

Mail id: drchandrajag [at] gmail.com 


\subsection{Isolation of RNA}

Balanus amphitrite RNA was isolated from adult barnacles by using Qiagen's DNA/RNA kit. RNA was further treated with RNase free DNase (Ambion, USA) in order to remove any contaminating genomic DNA. The quality of the extracted RNA was checked by formaldehyde agarose gel electrophoresis and concentration was measured by Nanodrop ND-1000 spectrophotometer.

\subsection{Preparation of cDNA library of adult barnacle, Balanus amphitrite}

cDNA library of adult barnacles, Balanus amphitrite was constructed in In-Fusion SMARTer Directional cDNA Library Construction Kit (Clontech Laboratories, Inc., USA) as per manufacturer's instructions. Briefly, double stranded cDNA was synthesized from 180ng of total RNA. SMART(er) technology enables full-length cDNA synthesis from nanograms of poly A+ or total RNA. After long-distance PCR (LD-PCR) of the ds cDNA, the amplified product was cloned in PSMART2IFD vector and transformed in E. coli by electroporation. Transformants were selected on ampicillin containing LB agar plates. Plasmids were isolated from recombinant clones in order to identify cloned genes.

\subsection{Sequencing of cDNA clones}

Plasmid DNA was isolated from recombinant clones by Plasmid isolation kit (Invitrogen). The plasmid DNA was checked in agarose gel electrophoresis and insert was sequenced with vector DNA specific flanking primers (F: 5'TCACACAGGAAACAGCTATGA-3' and R: 5'CCTCTTCGCTATTACGCCAGC-3') in both the direction by Sanger's dideoxy chain termination method.

\subsection{Bioinformatics analysis}

The nucleotide sequence was analysed by ORF finder (http://www.ncbi.nlm.nih.gov) in order to predict the ORF. The sequence comparison of predicted protein was conducted through database searches using pBLAST program (http://www.ncbi.nlm.nih.gov). Signal sequence was predicted by SignalIP 4.0 tool available at www.cbs.dtu.dk/services/SignalP/ [11]. Hydropathy plot was generated using deduced amino acid sequence with Expasy tool available at http://web.expasy.org/protscale/ [12]. For phylogenetic analysis, alignment of Balanus amphitrite cuticle protein with other closely related sequences was carried out with phylogeny.fr software using default parameters [13]. A phylogenetic tree was constructed with the help of aligned sequences using neighbour-joining method [14].

\section{RESULTS AND DISCUSSION}

The sequencing and analysis of Balanus amphitrite cDNA clone BCP-2, identified a complete gene sequence encoding for putative cuticular protein. Out of 100 randomly sequenced cDNA clones, two clones of BCP-2 were observed.
ORF prediction with 'ORF finder' predicted an ORF of 102 amino acids in BCP-2 DNA sequence. The deduced amino acid sequence showed that this protein is highly rich in alanine (22\%). Apart from alanine, valine and proline were also found abundant amounting together to $23 \%$ of total amino acids of this protein. The amino acid sequence of BCP-2 is found predominantly hydrophobic as observed in the hydropathy plot (Fig. 2). Hydropathic analysis of BCP-2 showed presence of two hydrophobic regions surrounding the central hydrophilic region (Fig. 2).

Similar observation was made with cuticular protein, TMLPCP-22 of Tenehrio molitor [8]. The first 16 amino acids of BCP-2 at N-terminus probably correspond to the signal sequence as a cleavage site was observed between 16 and 17 residues with SignalIP 4.0. The BCP-2 protein sequence lacked the presence of characteristic $R \& R$ motif in it, but instead showed presence of three $\mathrm{AAP}(\mathrm{V} / \mathrm{L})$ motifs at the $\mathrm{C}$-terminal end. It has been observed that many cuticular proteins lacks $R \& R$ motif and have other motifs that have been recently identified [8]. AAP(V/L) motifs have been found in many cuticular proteins from Locusta migratoria, Drosophila melanogaster, Tenebrio molitor, Bombyx mori and Manduca sexta, $[8,15-18]$ all grouped under the class Insecta. The presence of $\mathrm{AAP}(\mathrm{V} / \mathrm{L})$ containing cuticular protein has been reported from a crustacean, Cancer pagurus (crab) by direct sequencing of the exoskeleton proteins. Apart from this, AAP $(\mathrm{V} / \mathrm{L})$ containing putative cuticular proteins have been even identified from Marsupenaeus japonicas (Class: Crustaceans) as per the entries from CuticleDB. AAP(V/L) containing cuticular proteins are characteristic of hard cuticles. In case of BCP-2, all three motifs were found preceded by Tyrosine and Histidine residues (Fig. 3). Histidine residues in cuticular proteins are known to interact with catecholamines during sclerotization [19]. The tyrosine residues have been implicated in dityrosine bridge formation during sclerotization process in insect resilin [20] and in nematode cuticle protein CUT-2 [21]. Hence, presence of these repeating residues in BCP-2 along with $\mathrm{AAP}(\mathrm{V} / \mathrm{L})$ motifs may probably indicate its importance in sclerotization. BLAST analysis of BCP-2 amino acid sequence showed homology with cuticular proteins of domestic silkworm Bombyx mori, monarch butterfly Danaus plexippus and swallowtail butterfly, Papilio xuthus. Phylogenetic tree of BCP-2 with related cuticular proteins from other organisms was constructed with neighbor joining method using phylogeny.fr (Fig. 4). The cuticular proteins of Bombyx mori were found to form a cluster while three Danaus plexippus cuticular proteins formed another cluster (Fig. 4). One Danaus plexippus cuticular protein (Accession No. EHJ770911) was found clustered with Bombyx mori group rather than clustering with Danaus plexippus proteins. BCP-2 did not cluster with these two groups but showed its relatedness to Bombyx mori cuticular proteins as compared to Danaus plexippus cuticular proteins. The BLAST analysis of BCP-2 also shows better score with Bombyx mori cuticular proteins as compared to Danaus plexippus proteins, thus further authenticating the results of phylogenetic tree construction. 


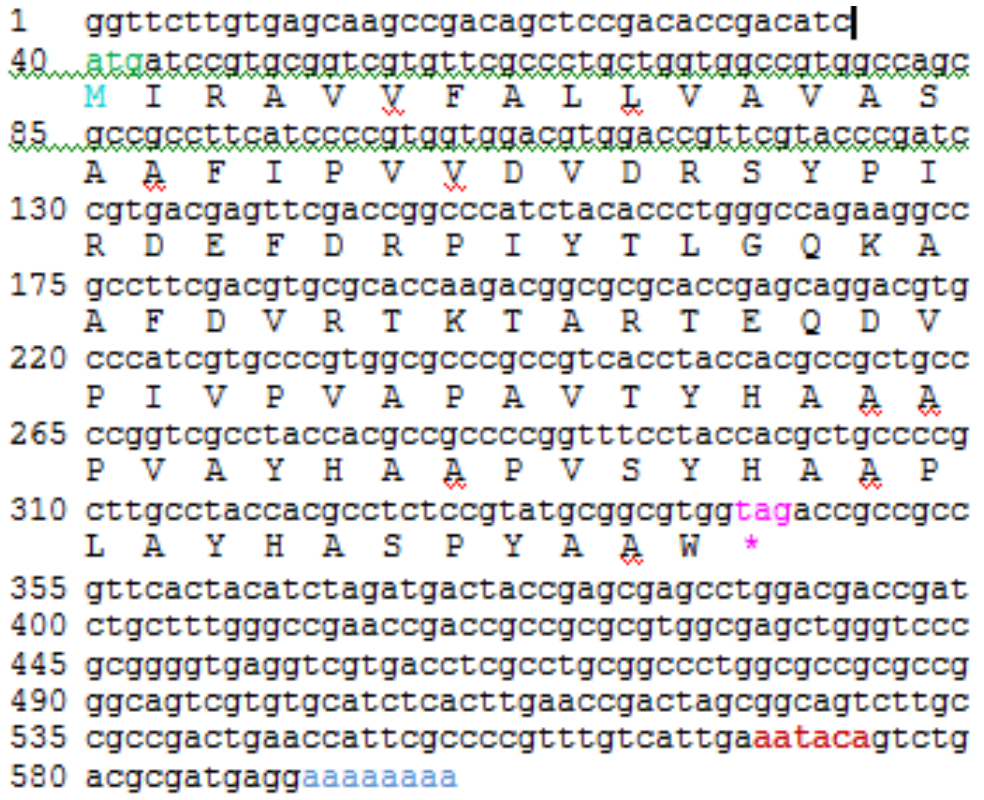

Fig. 1: cDNA sequence encoding Balanus amphitrite culticular protein BCP-2. Open reading frame was predicted with ORF finder (http://www.ncbi.nlm.nih.gov). Start codon (atg), stop codon (tag), putative poly adenylation site (aataca) are marked.

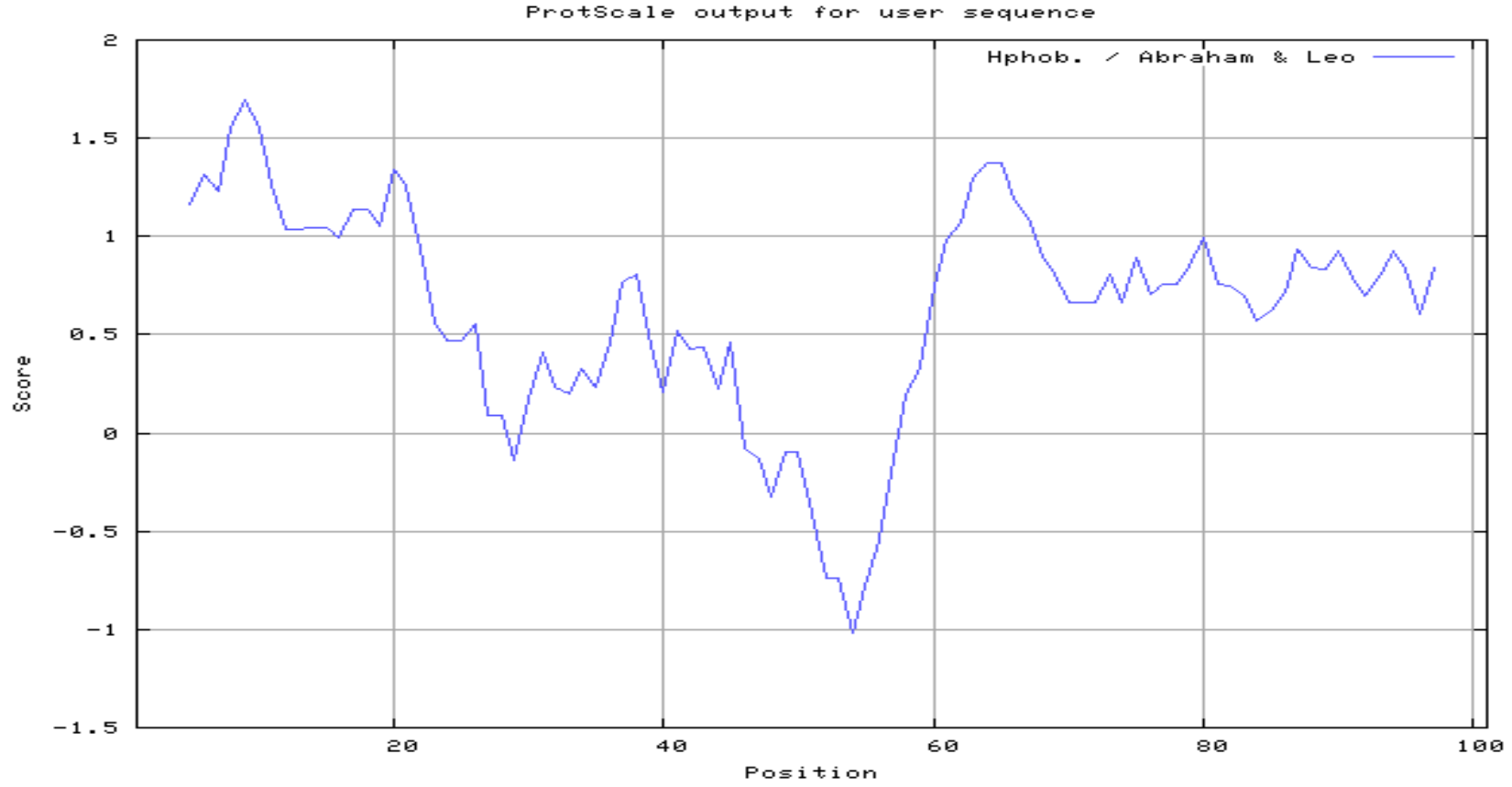

Fig. 2: Hydropathy plot of BCP-2. The plot was constructed by the method of Abraham and Leo (1987) using online tool available at Expasy (web.expasy.org/protscale/).

\section{$\begin{array}{llllllllll}71 & Y & H & \text { A } & \text { A } & \text { A } & \text { P } & \text { V } & \text { A }\end{array}$ \\ 79 Y $\mathrm{H}-\mathrm{A}$ A $\mathrm{P}$ V $\mathrm{S}$ \\ 86 Y H - A A $P$ L A \\ $93 \mathrm{Y} H-\mathrm{A} S \mathrm{P}$ Y A}

Fig. 3: Sequence comparison of internal repeats present in BCP-2. Note the presence of tyrosine and histidine residues preceding AAP(V/L) sequence. 
Interestingly, cuticular protein of swallowtail butterfly, Papilio xuthus (Accession no. BAG30763) did not cluster with cuticular proteins of monarch butterfly, Danaus plexippus; rather it was found distantly related to it as compared to BCP-2 (Fig. 4).

As per our knowledge this is the first report of Balanus amphtrite cuticular protein containing AAP(V/L) motif in it. As cuticular proteins of insects and crustaceans are known to form exoskeletons with varying mechanical properties, in future, engineering of these proteins for manufacturing biomaterials for various applications cannot be ruled out. Further identification and characterization of other cuticluar proteins from Balanus amphitrite and other crustaceans would help cataloging of these proteins systematically.

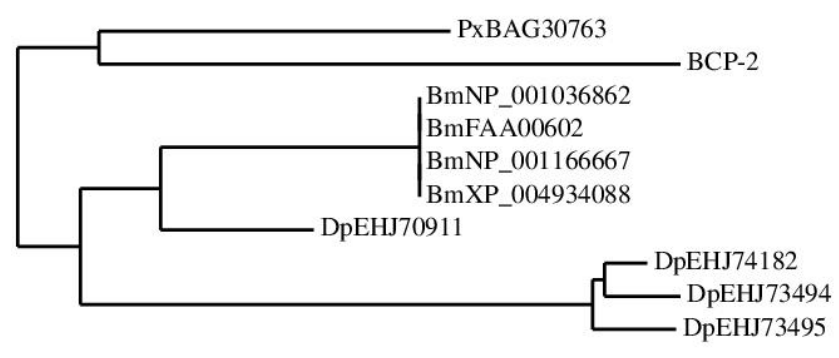

0.2

Fig. 4: Phylogenetic tree showing relationship of BCP-2 with other cuticular proteins from Bombyx mori (Accession nos. NP_001036862, FA A00602, NP_001166667, XP_004934088), Danaus plexippus (Accession nos. EHJ73494, EHJ74182, EHJ73495, EHJ770911), and Papilio xuthus (Accession no. BAG30763). Phylogenetic tree was constructed using Phylogeny.fr (http://www.phylogeny.fr/alacarte) with neighbour joining method.

\section{ACKNOWLEDGEMENT}

We are grateful to Director, Dr H. S. Hastak, NMRL, Ambernath for his kind support.

\section{REFERENCES}

1. Futahashi, R., Okamoto, S., Kawasaki, H., Zhong, Y-S., Iwanaga, M., Mita, K., \& Fujiwara, H. Genome-wide identification of cuticular protein genes in the silkworm, Bombyx mori. Insect Biochemistry and Molecular Biology. 2008; 38:1138-1146.

2. Roberts, P. E., \& Willis, J. H. The cuticular proteins of Tenebrio molitor. I. Electrophoretic banding patterns during postembryonic development. Dev Biol. 1980; 75:59-69.

3. Chihara, C.J., Silvert, D.J., \& Fristrom, J.W. The cuticle proteins of Drosophila melanogaster: stage specificity. Dev Biol. 1982; 89:379388.

4. Sridhara, S. Cuticular proteins of the silkmoth Antheraea polyphemus. Insect Biochemistry. 1983;13: 665-675.

5. Rebers, J. E., \& Riddiford, L. M. Structure and expression of a Manduca sexta larval cuticle gene homologous to Drosophila cuticle genes. J Mol Biol. 1988; 203:411-423.
6. Rebers, J. E., \& Willis, J. H. A conserved domain in arthropod cuticular proteins binds chitin. Insect Biochem Mol Biol. 2001; 27:229-240.

7. Togawa, T., Nakato, H., \& Izumi, S. Analysis of the chitin recognition mechanism of cuticle proteins from the soft cuticle of the silkworm, Bombyx mori. Insect Biochem Mol Biol. 2004; 34: 1059-1067.

8. Rondot, I., Quennedey, B., Courrent, A., Lemoine, A., \& Delachambre J. Cloning and sequencing of a cDNA encoding a larval-pupal-specific cuticular protein in Tenebrio molitor (Insecta, Coleoptera). Eur. J. Biochem. 1996; 235: 138-143.

9. Apple, R.T., \& Fristrom, J.W. 20-Hydroxyecdysone is required for, and negatively regulates, transcription of Drosophila pupal cuticle protein genes. Dev. Biol. 1991; 146: 569-582.

10. Nøhr, C., Andersen, S.O. Cuticular proteins from fifth instar nymphs of the migratory locust, Locusta migratoria. Insect Biochem. Mol Biol. 1993; 23:521-531.

11. Petersen, T. N., Brunak, S., Heijne, G. V. \& Nielsen H. SignalP 4.0: discriminating signal peptides from transmembrane regions. Nature Methods. 2011; 8:785-786.

12. Abraham D. J., \& Leo A.J. Hydrophobicity (delta G1/2 cal). Proteins: Structure, Function and Genetics. 1987; 2:130-152.

13. Dereeper, A., Guignon, V., Blanc, G., Audic, S., Buffet, S., Chevenet, F., Dufayard, J.F., Guindon, S., Lefort, V., Lescot, M., Claverie, J.M., \& Gascuel, O. Phylogeny.fr: robust phylogenetic analysis for the nonspecialist. Nucleic Acids Res. 2008; 1:36.

14. Saitou, N., \& Nei, M. The neighbor-joining method: a new method for reconstructing phylogenetic trees. Mol Biol Evol. 1987;4: 406-25.

15. Andersen, S. O. Studies on proteins in post-ecdysial nymphal cuticle of locust, Locusta migratoria, and cockroach, Blaberus craniifer. Insect Biochem Mol Biol. 2000; 30:569-77.

16. Charles, J.P., Chihara, C., Nejad, S., \& Riddiford, L.M. Identification of proteins and developmental expression of RNAs encoded by the 65A cuticle protein gene cluster in Drosophila melanogaster._Insect Biochem. Mol. Biol. 1998; 28: 131--138.

17. Andersen, S.O., Rafn, K., \& Roepstorff, P. Sequence studies of proteins from larval and pupal cuticle of the yellow meal worm, Tenebrio molitor. Insect Biochem. Mol. Biol. 1997; 27: 121-131.

18. Mathelin, J., Quennedey, B., Bouhin, H., Delachambre, J. Characterization of two new cuticular genes specifically expressed during the post-ecdysial molting period in Tenebrio molitor. Gene 1998; 211: 351-359.

19. Andersen, S.O., Højrup, P., \& Roepstorff, P. Insect cuticular proteins. Insect Biochem. Mol. Biol. 1995; 25, 153-176.

20. Andersen, S. O. The cross-links in resilin identified as dityrosine and trityrosine. Biochim biophys acta. 1964; 93:213-5.

21. Lassandro, F., Sebastiano, M., Zei, F., \& Bazzica-lupo, P. The role of dityrosine formation in the crosslinking of CUT-2, the product of a second cuticlin gene of Caenorhabditis elegans. Molecular and Biochemical Parasitology. 1994; 65:147-159.

\section{How to cite this article:}

Chandrakant B. Jagtap, Om Kinker Tiwari, Pradeep Kumar. cDNA cloning and analysis of alanine-rich cuticle protein gene of common fouling barnacle, Balanus amphitrite. J App Biol Biotech. 2015; 3 (03): 028-031. 